\section{hommes}

\section{Hommes \& migrations}

Revue française de référence sur les dynamiques

migratoires

$1304 \mid 2013$

Frontières

\title{
Cartographie de la frontière et topographie clandestine
}

\section{Daniel Veron}

\section{(2) OpenEdition \\ Journals}

\section{Édition électronique}

URL : http://journals.openedition.org/hommesmigrations/2630

DOI : 10.4000/hommesmigrations.2630

ISSN : 2262-3353

\section{Éditeur}

Musée national de l'histoire de l'immigration

\section{Édition imprimée}

Date de publication : 1 octobre 2013

Pagination : 19-25

ISBN : 978-2-919040-24-7

ISSN : $1142-852 X$

\section{Référence électronique}

Daniel Veron, «Cartographie de la frontière et topographie clandestine », Hommes \& migrations [En ligne], 1304 | 2013, mis en ligne le 01 janvier 2017, consulté le 20 avril 2019. URL : http:// journals.openedition.org/hommesmigrations/2630; DOI : 10.4000/hommesmigrations.2630 
Sur un piquet de grève à Nanterre en février 2010.

En arrière-plan, le quartier d'affaires de La Défense.

๑) Nicolas Romero 


\section{CARTOGRAPHIE DE LA FRONTIĖRE ET TOPOGRAPHIE CLANDESTINE}

par DANIEL VERON, doctorant en sociologie, université Paris-Ouest Nanterre, laboratoire IDHE (UMR 8533)

La frontière n'est pas seulement un espace géographique à franchir, elle est également devenue une institution. Les migrants sans papiers échafaudent des tactiques de contournement de la frontière et de ses avatars que sont les centres de rétention, les procédures de demande d'asile, etc. Ils tentent de mettre à profit le moindre interstice, la moindre brèche face aux murs que représentent les déploiements législatifs et symboliques de la frontière. Leurs parcours s'inscrivent de ce fait dans une topographie clandestine qu'ils contribuent à dessiner.

\section{Surveillance et détention, les clés de la frontière}

Pour les migrants ayant réussi à déjouer les déploiements frénétiques d'énergie visant à les tenir en dehors des territoires nationaux des pays dits "développés", la frontière ne s'efface pas pour autant. Bien au contraire, l'“institution frontière ${ }^{1 ”}$, à la géographie complexe et mouvante, semble aujourd'hui connaître dans les pays occidentaux une diffusion et une crispation sans précédent: "La frontière géographique n'est qu'un moment d'une chaîne d'obstacles à l'intérieur d'un même pays"." Pour ceux qui sont désormais des "sans-papiers ${ }^{3}$ ", l'interface avec l'institution frontière, qui connaît à la fois une externalisation ${ }^{4}$ et une expansion à l'intérieur du territoire national, est conditionnée par leur "déportabilité5". Les déclinaisons du dispositif d'enforcement de cette institution sont multiples, et la violence induite multiforme. En Europe, la police en est sans doute la manifestation la plus évidente, car en première ligne dans cette "chasse aux sanspapiers $^{6 "}$ : contrôles, quadrillage et surveillance systématiques et organisés de l'espace national. Elle a pour corollaire les infrastructures de détention, clé 
de voûte et expression la plus brutale du dispositif policier engagé à leur encontre. L'institution frontière se manifeste également dans les diverses administrations avec lesquelles les sans-papiers sont contraints de dialoguer : la préfecture bien sûr, mais aussi les différents guichets des caisses d'assurances sociales, des services bancaires ou postaux, et jusqu'aux lieux de travail. L'institution frontière colonise le monde vécu ${ }^{7}$ des sans-papiers, et plus largement des migrants. Pour autant, ce monde vécu est aussi - et peut-être surtout - un monde de résistances, d'ajustements, de ruses, de dérobades, autrement dit "un univers tactique $e^{8 ”}$. À partir d'une analyse comparative menée sur trois terrains (Paris, Buenos Aires et Montréal), il s'agit de s'intéresser à la dialectique étroite et complexe qui se donne à lire entre deux logiques hétérogènes : celle de l'institution qui entend avant tout gérer des corps ${ }^{9}$, et celle des hommes et des femmes qui quotidiennement composent face à un dispositif répressif multiforme. Ces tactiques se fondent sur un savoir pratique des déploiements de l'institution frontière. Ce savoir, qui procède d'une expérience "par le bas" de la cartographie de la frontière, esquisse les lignes de fuite d'une topographie de la clandestinité.

\section{Le marquage des corps}

"L'Europe a développé une technique, ils centralisent les empreintes pour connaître toutes les demandes, donc on doit développer nos propres techniques", raconte un migrant érythréen aux alentours de Calais face à la caméra de Sylvain Georges ${ }^{10}$, tout en se brûlant le bout des doigts à l'aide d'une vis chauffée à blanc. La "technique" européenne qu'il évoque fait référence au fichier Eurodac, qui centralise toutes les données administratives - et biométriques relatives aux demandes d'asile et de visas, mais aussi aux contrôles d'entrée, arrestations, etc. En vertu de l'accord européen dit "Dublin II", qui stipule entre autres que le pays d'entrée sur le territoire européen est celui où la demande d'asile doit être déposée, ces migrants, en cas d'échec de leur tentative de passage vers l'Angleterre, risquent un renvoi vers le premier pays "sûr" par lequel ils ont transité11. C'est d'abord le corps lui-même qui apparaît comme un lieu de réalisation du pouvoir, ou de son "échappée 12". "Pour refuser d'embarquer, un mec a eu une idée incroyable. Il s'est chié dessus. Il s'est tout étalé sur lui. Ils n'ont pas pu l'expulser. Ils l'ont ramené au centre. Le lendemain, ils sont venus le chercher. Ils l'ont attaché avec du scotch et ils l'ont enroulé dans du film plastique. Ils l'ont pris et ils l'ont expulsé comme ça ${ }^{13}$." Ce témoignage rejoint tous ceux qui évoquent les diverses mutilations - ingestion de lames de rasoir, veines tailladées, pendaison - qui ont cours dans les centres de rétention administrative (CRA). Ces actes de retournement de la violence sur soi sont mus non tant par le désespoir, que par la recherche d'un "juste assez" de mutilations pour échapper à l'expulsion. Corps mutilés, suffisamment déformés pour déjouer l'institution policière, "ingéniosités du faible pour tirer parti du fort ${ }^{14}$ ", les tactiques déployées par les migrants en situation d'illégalité migratoire ne se réduisent pourtant pas à une liminalité dont la forme pure se rapprocherait de ce que Giorgio Agamben a conceptualisé comme la "vie nue ${ }^{15}$ ". Si cette radicalité de l'interaction avec la frontière est toujours potentiellement présente, la vie clandestine s'organise et se structure autour de ce qui constitue l'économie de la clandestinité. 
Simbo, gréviste et sans-papiers, montre un document des impôts lui annonçant le versement de la prime pour l'emploi. @ Nicolas Romero

\section{Les institutions bâtardes de la vie clandestine}

Comme le suggère Sébastien Chauvin ${ }^{16}$, ces formes organisées renvoient à ce que Everett Hughes nomme les "institutions bâtardes ${ }^{17 "}$. On pense à la figure du "passeur", bien sûr, dont les rôles et les formes varient beaucoup : du simple individu, luimême migrant, possédant simplement un semblant de savoir sur la route à emprunter, offrant pour quelques écus d'emmener avec lui des compagnons de voyage, à l'entreprise d'un village ou peut- être d'une fratrie ayant investi dans la construction d'une embarcation, jusqu'au réseau criminel plus ou moins organisé, dont les modes de fonctionnement et de rétribution de ses services se rapprochent des cas de traite ${ }^{18}$, en passant par le marché noir des visas ${ }^{19}$.

Viennent ensuite les accès parallèles au marché de l'emploi : travail au noir, vente à la sauvette, jusqu'à l'accès au marché du travail légal, souvent via l'intérim ou l'agence de travail journalier - là aussi à penser plutôt comme un continuum d'institutions dont la porosité entre les différents niveaux de formalité est forte. "Vous dites fausses cartes, mais pour nous c'est des vrais papiers", m'explique Moussa, Malien régularisé en 2009 après huit années 
passées en France sans papiers. Quelle différence en effet, sachant qu'un tel 'marché

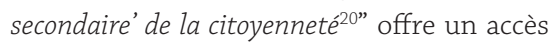
- certes partiel et limité - au travail ?

L'enjeu du logement suscite lui aussi des tactiques multiples. C'est "l'habiter des interstices $^{21 "}$ d'abord, qui implique de jouer avec les interstices urbains, usant de rebuts, et dont la "jungle" de Calais est sans doute la forme idéale typique. "Alors, sans s'en sortir encore, habiter la 'jungle', en faire un lieu convivial, accueillant, reposant, tout en la maintenant dans sa précarité évidemment, est un art de survivre qu'apprennent les exilés22."

Le foyer, "village africain à la verticale" selon Djiby, migrant sénégalais en France depuis 2000, est aussi une institution bâtarde dans laquelle les sans-papiers puisent nombre de ressources ${ }^{23}$ : logement, bien sûr, dans des formes de sous-locations informelles, mais aussi accès au marché des faux papiers, à l'information, voire à l'organisation (les foyers jouent un rôle majeur dans la grève des travailleurs sans papiers).

Dans ces lieux de la vie clandestine circulent des savoirs pratiques, empiriques ${ }^{24}$. S'y élaborent et s'y transmettent la cartographie de l'institution frontière et les tactiques de déplacement qu'implique l'illégalité migratoire.

\section{Cartographier la frontière}

La question de la spatialité est centrale. Une spatialité concrète - avec la nécessité d’aménager des espaces de refuge -, mais aussi une spatialité plus abstraite, cognitive - celle de la cartographie de l'institution frontière. Si on cherche à rendre compte des subjectivités clandestines, ainsi que l'horizon cognitif des migrants illégalisés, il faut

Le piquet de grève de Nanterre, sur lequel les grévistes ont vécu jour et nuit pendant neuf mois. (C) NicolAs ROMERO

saisir l'enjeu de l'élaboration de cette cartographie. Parlant de son expérience de l'administration canadienne, un migrant somalien évoque : "Un couloir sans fin où aucune porte ne serait ouverte".

Les organisations de soutien jouent un rôle important. Une de leurs fonctions, dans la quotidienneté de l'accompagnement face aux méandres de l'administration et à ses pièges, est précisément de baliser l'espace du possible : énoncer les droits, pointer les impossibilités, aménager des espaces de refuge. Autrement dit, là aussi, cartographier l'institution frontière, ses murs et ses remparts, ses voies obligées comme ses chemins de traverse.

Au Canada, un moment crucial pour les déboutés de l'asile est celui de la réponse de l'ultime recours, l'Examen des risques avant renvoi (ERAR) ${ }^{25}$. Convoqués dans les locaux de l'Agence des services frontaliers du Canada, ils sont interrogés sur leur volonté de partir dans un délai de quelques semaines. La réaction spontanée est souvent de chercher à plaider sa cause, attitude systématiquement inter-

20. Sébastien Chauvin, Les Agences de la précarité. Journaliers à Chicago, op. cit. 21. Michel Agier, Sara Prestianni, "Je me suis réfugié là !" Bords de routes en exil, Paris, Donner Lieu, 2011. 22. Ibid. 23. Voir aussi Anaïk Pian qui décrit la fonction ressource des foyers de migrants au Maroc. Anaïk Pian, Aux nouvelles frontières de l'Europe. L'aventure incertaine des Sénégalais au Maroc, Paris, La Dispute, 2009. 24. Daniel Veron, “Quand les sans-papiers prennent la parole. Espaces d'interlocution et énonciation du tort”, in Variations, $n^{\circ}$ 18, 2013. 25. L'Examen des risques avant renvoi est un dernier examen, administratif, des éléments du dossier du demandeur (et éventuellement de nouvelles pièces) avant la "déportation" - c'est le terme utilisé au Québec - vers le pays d’origine. Si l'examen établit un risque réel pour l'intégrité de la personne, celle-ci obtient automatiquement la résidence. Mais le taux de réponses positives est extrêmement faible. 
prétée par l'administration comme l'intention de se soustraire à l'ordre de renvoi. La personne est alors placée en détention jusqu’à l'expulsion.

Aussi, un des "trucs" à savoir, c'est qu'à l'annonce du refus d'un ERAR, il faut faire montre de la meilleure volonté et se plier à toute injonction de l'agent en face de soi. L'enjeu est, là encore, de garder des marges de manœuvre, de conserver la possibilité d'un "choix", et ainsi d'organiser soit son départ soit une entrée dans la vie clandestine. Dans ce dernier cas, c'est un tout nouveau paysage qui s'annonce : il s'agit désormais d'apprendre, en creux des reliefs de l'institution, les dynamiques d'une topographie clandestine.

\section{Topographie clandestine}

À Montréal, une des orientations choisies par les organisations de soutien aux "sans-statut" est à ce titre intéressante. L'organisation Solidarité sans frontières mène une campagne "pour une cité sans frontières ${ }^{26 "}$ en direction des écoles, des hôpitaux, des refuges pour femmes et de divers organismes communautaires qui vise, à défaut d'un changement dans les politiques migratoires, l'effectivité de l'accès aux droits pour les personnes sans statut ${ }^{27}$. Soit l'institutionnalisation d'espaces de refuge, c'est-à-dire travailler à consolider et à étendre ces espaces tactiques d'autonomie où la circulation devient possible pour les migrants illégalisés.

Cette circulation aux "trajectoires indéterminées, apparemment insensées parce qu'elles ne sont pas cohérentes avec l'espace bâti, écrit et préfabriqué où elles se déplacent ${ }^{28}$, dessine précisément cette topographie de la clandestinité : espaces d'autonomie, zones de danger, chemins de traverse. Or "ces 'traverses' demeurent hétérogènes aux systèmes où elles s'infiltrent et où elles dessinent des ruses d'intérêts et de désirs différents. Elles circulent, vont et viennent, débordent et dérivent dans un reliefimposé, mouvances écumeuses d'une mer s'insinuant parmi les rochers et dédales d'un ordre établi ${ }^{29 "}$.

Parce que clandestine, cette topographie se place en partie sous le sceau du secret. Aïcha, migrante ivoirienne ayant passé quatre ans dans la clandestinité au Québec, m'interroge longuement, avant que nous ne commencions l'entretien, sur mon travail, sur ses finalités et surtout sur ses futurs lecteurs. Elle s'inquiète de la confidentialité des informations qu'elle est susceptible de me dévoiler sur les tactiques de vie sans statut, affichant ainsi une conscience aiguë de l'importance de protéger la topographie clandestine. La même préoccupation motive Solidarité sans frontières dans le choix de ne diffuser que de la main à la main le "guide de survie" publié à l'usage des personnes sans statut. La morphologie de ces espaces tactiques
La question de la spatialité est centrale.

Une spatialité concrète - avec la nécessité d'aménager des espaces de refuge -, mais aussi une spatialité plus abstraite, cognitive celle de la cartographie de l'institution frontière. gré des évolutions législatives et des pratiques administratives ou policières - mises à jour cartographiques -, mais aussi au gré des tactiques de résistance qui ouvrent des possibles - évolutions topographiques -, et dont l'institution frontière se saisitrégulièrementpourfaireévoluerses dispositifs de contrôle.

\section{Détournement d'une institution légitime : I'asile}

Si la vie des migrants illégalisés puise son organisation dans des institutions bâtardes qui lui sont propres, créant ainsi des espaces d'autonomie, il arrive aussi que des institutions légitimes soient 
détournées de leur usage officiel. La fermeture des voies de migration a entraîné un usage tactique de la demande d'asile, produisant effectivement de "faux" demandeurs au sens de l'institution. Le cas des migrants mexicains au Canada est à ce titre éclairant. "À cette époque, cétait la mode de l'asile", me commente à l'automne 2011 Joël, migrant mexicain au Canada depuis trois ans. En 2000, 1317 citoyens mexicains demandaient l'asile. Ils étaient 9319 en 2009, soit une augmentation de plus de $700 \%$ et une progression annuelle moyenne de l'ordre de $20 \%$ (l'augmentation étant assez linéaire) $)^{30}$. "Beaucoup ont profité de ça, du refuge ici au Canada, et ils ont commencé à ouvrir des agences [au Mexique] où ils te donnaient des informations. Ils nous prenaient 1300 dollars pour venir ici, sans compter le billet d'avion", raconte Julia, mexicaine au Canada depuis trois ans. Depuis la signature de l'Accord de libre-échange de l'Amérique du Nord (ALENA) en 1994, les ressor-

L'administration interprète

les usages tactiques de la cartographie

institutionnelle comme de la malhonnêteté, du vice ou de l'immoralité. tissants mexicains n'avaient, jusqu'à une date récente, pas besoin de visa pour entrer sur le territoire canadien. Une fois entrés, certains d'entre eux déposaient une demande d'asile. Ils se voyaient alors fournir une carte d'accès au système de santé ainsi que, sur demande, un permis de travail. Autrement dit, un statut légal pour les deux à quatre ans que dure l'instruction de leur dossier. Plusieurs dizaines de milliers de candidats à la migration auront vu dans la possibilité d'un départ pour le Canada une alternative à la route vers les États-Unis. L'immense majorité déchantera rapidement : de $26 \%$ en 2000 , le taux d'acceptation des demandes de refuge tombe à 8 \% en 2009.
Face à ce qui est décrit comme des "abus" et un "fardeau" pour le système de demande d'asile canadien $^{31}$, le gouvernement décide, en juillet 2009, de colmater cette voie d'entrée et remet provisoirement en place l'obligation de visa pour les Mexicains. L'effet est immédiat : en 2010, le nombre de demandeurs d'asile mexicains chute à 1321 ; ils seront 804 l'année suivante ${ }^{32}$.

\section{Du point de vue de l'institution}

Depuis quelques années, l'Argentine connaît une immigration faible mais constante en provenance du Sénégal. Transitant par le Brésil, pays pour lequel ils obtiennent (au cours des années $2000 \mathrm{du}$ moins) relativement facilement un visa, les migrants sénégalais entrent ensuite de façon clandestine en Argentine par voie terrestre. Les quelques milliers de Sénégalais à suivre cette voie, en grande majorité des hommes, déposent à leur arrivée en Argentine une demande de refuge, seule manière d'obtenir un titre de séjour, même précaire $^{33}$. Pour l'essentiel des Mourides, leur intégration communautaire est forte et leur activité économique très homogène (vente ambulante). Laccès au logement étant quasiment impossible, ils sont contraints de se tourner vers les marchands de sommeil ou de vivre en squat. Enfin, aucune perspective de régularisation ne s'offre à eux, si ce n'est le mariage.

Alors, quand est découvert un immeuble squatté par trente Sénégalais, ou lorsque cent trente mariages sont officiés le même jour, du point de vue de la Direction nationale des migrations, il s'agit d'un cas de réseau de migration criminel, voire d'un cas de traite des personnes, comme me

30. Source : Commission de l'immigration et du statut de réfugié au Canada. Voir Annie Lapalme, "Citoyenneté, mondialisation et migration internationale. Les réfugiés et demandeurs d'asile mexicains à Montréal", mémoire de maîtrise en géographie, université de Montréal, 2013. 31. Selon les propos du ministre de l'Immigration Jason Kenney, communiqué du 13 juillet 2009. 32. En juin 2012, le gouvernement Harper a fait voter une loi (C-31) de réforme du système d'immigration ouvrant une procédure "accélérée" d'examen des demandes d'asile pour les pays dits "sûrs", dont le Mexique (pays offrant pourtant des taux d'homicides parmi les plus élevés au monde, ce qui n’a d'ailleurs pas échappé à un autre ministère canadien, celui des Affaires étrangères, qui déconseille à ses ressortissants de se rendre sur près de la moitié du territoire mexicain). Selon le Conseil canadien pour les réfugiés, cette procédure rend impossible une défense correcte des demandeurs. Carte d'accès à la santé et permis de travail ne sont plus octroyés dans le cadre de cette procédure. 33. Ce titre est justement appelé communément

"la precaria". Il est renouvelable tous les 3 mois pendant la durée d'instruction du dossier, qui peut prendre plusieurs années. Pour les Sénégalais, le refus est quasi systématique. 
l'explique le président de la fondation Citoyens du monde, organisation de soutien aux migrants. "Eux disent qu'il y a une mafia. J'ai eu l'occasion de discuter avec eux, et ils m'ont même interpellé en m'interrogeant sur mon intérêt personnel, s'il n'y avait pas un intérêt économique."

L'administration interprète les usages tactiques de la cartographie institutionnelle comme de la malhonnêteté, du vice ou de l'immoralités ${ }^{3}$. L'Argentine a depuis exigé de son voisin brésilien qu'il revoie ses politiques de visa en direction des Sénégalais et, en 2009, a ôté la compétence de certification des documents officiels exigés pour toute demande de séjour à son ambassade au Brésil. Les Sénégalais doivent désormais légaliser leurs documents... au Nigéria. La topographie argentine étant devenue de plus en plus impraticable, les migrants sénégalais s'en retournent vers le Brésil. "Ça fait un an que le Brésil donne des papiers à tous ceux qui avaient un visa. Moi j’ai des papiers brésiliens. Au moment où ils donnaient les papiers, j'y suis allé pour demander et je suis revenu. Maintenant, beaucoup partent là-bas", me raconte Cheick à l'automne 2010.

\section{La "brèche" du mouvement de grève des travailleurs sans papiers}

Dans cette dialectique qui pousse sans arrêt l'institution frontière à boucher les voies d'eau par où s'infiltrent les circulations clandestines, contraintes alors de trouver d'autres chemins, certaines dynamiques des "petits ${ }^{35}$ " peuvent parfois prendre des dimensions inattendues. En mai 2007, en France, un décret (2007-801) va venir bouleverser l'organisation de la topographie clandestine. La vérification en préfecture du titre de séjour présenté par un étranger pour toute nouvelle embauche est imposée.
Cette nouvelle disposition va entrainer une vague de licenciements de travailleurs sans papiers et, de manière générale, grandement compliquer leur accès au marché du travail. Alors que parallèlement la "loi Hortefeux" de novembre $2007^{36}$ ouvre la possibilité d'une régularisation "à titre exceptionnel", en vue de l'obtention d'un
Ce tour d'horizon fragmenté des tactiques des migrants illégalisés avait pour objectif de montrer l'importance qu'il y a à penser la frontière "par le bas". légitime dans la défense des droits du travail : le syn-

dicat. Cette rencontre, et les circonstances historiques dans lesquelles elle se déroule, mèneront à une mobilisation collective sans précédent ${ }^{37}$. Une "brèche ${ }^{38 "}$ est ouverte.

On découvre alors que les sans-papiers sont intégrés au monde du travail, beaucoup sont en CDI, dans des entreprises qui ont pignon sur rue. Ils construisent nos bâtiments, cuisinent nos plats, nettoient nos bureaux, gardent nos enfants. Ils payent aussi leurs impôts et cotisent à toutes les caisses d'assurance sociale.

Un coup de projecteur est donné sur tout un pan de cette topographie clandestine, au "point d'indistinction ${ }^{39 "}$ où formel et informel se mêlent et se confondent. Et où l'espace social devient un lieu de lutte.

Ce tour d'horizon fragmenté des tactiques des migrants illégalisés avait pour objectif de montrer l'importance qu'il y a à penser la frontière "par le bas". Cette plongée dans la "logique harmonieuse du chaos ${ }^{40 "}$ permet ainsi de saisir l'enjeu des rapports entre les migrants sans papiers et l'institution frontière : bricoler des lieux pour habiter le monde, construire des espaces d'autonomie, affiner les "arts de la résistance ${ }^{41}$ ". 